CERN-SL/95/77

18 September 1995

\title{
SINGLE-SHOT TEMPORAL MEASUREMENT OF SHORT X-RAY PULSES USING VERY FAST PHOTOCONDUCTIVE DETECTORS
}

\author{
M. Cuzin \\ CEA/LETI 17, rue des Martyrs, F-38054 Grenoble Cedex 9, France \\ E. Rossa \\ CERN, 1211 Geneva 23, Switzerland
}

\begin{abstract}
A report on the status of the development of picosecond photoconductive detectors is presented. The main application of these devices is the single-shot temporal measurement of picosecond X-ray, UV, and visible light pulses. We compare different materials, and describe the X-ray autocorrelator and the single-shot sampler developed in the LETI (Grenoble).
\end{abstract}

Submitted to the 9th International Workshop on Room Temperature Semiconductor $X$ and Gamma Ray Detectors Associated Electronics and Applications, Grenoble, France, 18-22 September 1995 
Cadmium telluride (CdTe) or gallium arsenide (GaAS) are well-known as good semiconductor materials for nuclear radiation detectors and electro-optic devices [1, 2]. Their high-charge carrier mobility provides an efficient charge collection, whilst their high atomic number provides high stopping power for gamma and X-rays. However, their use as very fast photoconductive detectors is not well recognized.

The charge collection is proportional to the energy deposited and the mobilitylifetime product of the electrons and holes. Since the lifetime of the carrier is one parameter, limiting the speed of the detector, we describe the polycrystalline photoconductive detector with a picosecond and subpicosecond lifetime. We have developed a single-shot $\mathrm{X}$-ray detector for the measurement of the intense synchrotron radiation emitted in the LEP (Large Electron-Positron collider) [3]-[5]. Thanks to the large deposited energy and fast electronics a single-shot measurement with a very small carrier lifetime is possible at a rate of $100 \mathrm{kHz}$.

A brief description of the X-ray source is followed by a presentation of the material selection and the auto-correlator electronics. Then we present the principle of a single-shot sampler of electric signals, which may be useful for some other X-ray, UV, or visible light sources.

\section{X-RAY SOURCE}

The first aim of using the detector [3]-[5] was to monitor the longitudinal distribution of electron bunches in the LEP by the intense X-ray synchrotron radiation they emit. When passing through the main bending magnets, the electrons (or positrons) radiate Xrays in the horizontal plane at a very small vertical emission angle. Thus, the vertical spot size is only $1 \mathrm{~mm}(\mathrm{FWHM})$ at the detector position. All the equipment is positioned in a recess of the vacuum chamber and irradiated through a beryllium window.

The continuous spectral distribution of the radiation peaks at around $100 \mathrm{keV}$ when the LEP is used at $45 \mathrm{GeV}$. The cut-off introduced by the Be window is $5 \mathrm{keV}$, and the incident energy per pulse is about $1.510^{-4} \mathrm{~J} / \mathrm{mm}^{2}$. At a repetition rate of 136350 pulses per second the mean power is $20.7 \mathrm{~W}$ per $\mathrm{mm}^{2}$. This is a very high power level; the detector is cooled down by water circulation in an aluminium radiator. The semiconductor quickly integrates a very high dose of radiation: for 1000 hours in the synchrotron radiation the dose is $8.910^{12} \mathrm{~Gy}$.

Therefore the choice of photoconductor is limited.

\section{MATERIAL SELECTION}

Free choice of materials involves the following considerations:

- The detector must be fast enough to give in a single shot the temporal shape of one $\mathrm{X}$-ray pulse of $30 \mathrm{ps}$ (nominal value of sigma).

- The semiconductor must survive a very high X-ray dose without losing its performances. An amorphous or polycrystalline photoconductor with only ohmic contact will be more resistant than the junction of a photodiode.

- The intense X-ray pulses induce a parasitic effect in insulator and polarization lines $[6,7]$. To improve the signal-to-noise ratio we have selected high $\mathrm{Z}$ material for the detector itself and low $\mathrm{Z}$ materials for the substrate, polarization lines, radiator and support.

Table 1 shows for gallium arsenide and cadmium telluride the very large range of carrier lifetimes which can be obtained by different technologies. BULK is massive 
crystal, MOCVD is metal organic chemical vapour deposition, and MBE is molecular beam epitaxy.

Table 1: Comparison of different photoconductors

\begin{tabular}{|c|c|c|c|c|c|c|}
\cline { 2 - 7 } \multicolumn{1}{c|}{} & \multicolumn{3}{c|}{ GaAs } & \multicolumn{3}{c|}{ CdTe } \\
\cline { 2 - 7 } \multicolumn{1}{c|}{} & BULK & MOCVD & MBE & BULK & MOCVD & MBE \\
\hline $\begin{array}{c}\text { Carrier lifetime } \\
\tau_{\mathrm{e}}\end{array}$ & $1 \mu \mathrm{s}$ & & $\begin{array}{c}0.8 \mathrm{ps} \\
0.15<<10\end{array}$ & $0.1 \mu \mathrm{s}$ & $<10 \mathrm{ps}$ & $0.8 \mathrm{ps}$ \\
\hline $\begin{array}{c}\text { Mobility } \\
\mu_{\mathrm{e}} \\
\left(\mathrm{cm}^{2} / \mathrm{V} \mathrm{s}\right)\end{array}$ & 8000 & $20<<55$ & 1000 & 100 & $<1$ \\
\hline $\begin{array}{c}\text { Dark resistivity } \\
\rho \\
(\Omega . \mathrm{cm})\end{array}$ & $>10^{7}$ & & $10^{5}<<10^{7}$ & $>10^{9}$ & $10^{7}<<10^{10}$ & $310^{7}$ \\
\hline
\end{tabular}

We have selected CdTe deposited by the MOCVD method because this material fulfils the above conditions, and the technology for it existed in the laboratory LETI in Grenoble when we began the project.

- The picosecond lifetime was possible — we have measured 5.7 ps lifetime for $12 \mu \mathrm{s}$ thickness of CdTe.

- The high Z (48 for $\mathrm{Cd}$ and 52 for $\mathrm{Te}$ ) provides a high stopping power. Moreover, the mobility is above $150 \mathrm{~cm}^{2} / \mathrm{V}$ s when the size of a single crystal reaches some microns in size.

- We have also verified that polycrystalline material is very resistant to extremely high doses of radiation [8].

\section{X-RAY AUTOCORRELATOR}

The aim of this detector is to monitor, in real time, the autocorrelation of the longitudinal bunch profile [3]-[5]. The electronics digitize a complete bunch profile every $11 \mu \mathrm{s}$, thus up to eight LEP bunches are continually analysed.

The light source is the intense X-ray synchrotron radiation emitted by the LEP main bending magnets. We have chosen to use X-rays because most of the energy is radiated in this range, and in the same vacuum tank we already have a vertical profile detector [8]. The latter is not diffraction-limited as is the usual visible light detector.

\subsection{Manufacture of the detector}

The whole circuit is placed on a thin quartz plate, $127 \mu \mathrm{m}$ thick, $50 \times 20 \mathrm{~mm}$. Folded delay lines (see Fig. 1) are produced by a precise vacuum deposit of aluminium. Fast CdTe photoconductors are connected to the delay line in a horizontal row at the level of the beam.

The same intense synchrotron radiation pulse simultaneously illuminates all semiconductors. The photoconductor $\mathrm{P}_{0}$, polarized with a DC bias voltage, sends through the line an electric signal $V(t)$ proportional to the incident light pulse $\mathrm{X}(\mathrm{t})$ (Fig. 1). This signal propagates in the two delay lines and biases the other semiconductors with various delays $\mathrm{T}_{\mathrm{i}}$. Any photoconductor Pi generates a current proportional to the product of the 
light pulse and of its delayed image $\mathrm{V}\left(\mathrm{t}+\mathrm{T}_{\mathrm{i}}\right)$. Thus the delivered charge $\mathrm{Q}_{\mathrm{i}}$,

$$
\mathrm{Q}_{\mathrm{i}}=\mathrm{k} \int_{-\infty}^{+\infty} \mathrm{X}(\mathrm{t}) \mathrm{V}\left(\mathrm{t}+\mathrm{T}_{\mathrm{i}}\right) \mathrm{dt},
$$

gives one point of the autocorrelation profile, corresponding to the delay $\mathrm{T}_{\mathrm{i}}$.

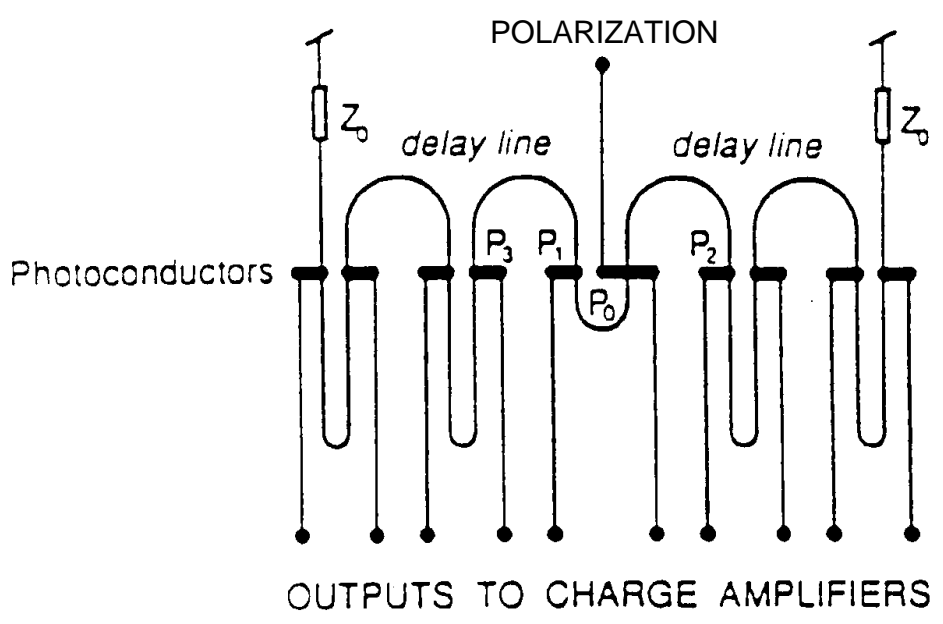

Figure 1: Principle of the autocorrelator.

The transmission of a very fast electric signal is critical only on the central delay line for a time equal to the photon bunch length. On the other hand the reading lines transmit charges only to the integrators outside the circuit. Since we have selected stripline technology, which is not dispersive, very short signals propagate on the short distance in the central delay line. Moreover the $127 \mu \mathrm{m}$ thick substrates in quartz or ceramic are transparent for the X-ray in the CERN application and the parasitic effects are minimized [3].

An example of final construction is shown in Fig. 2.

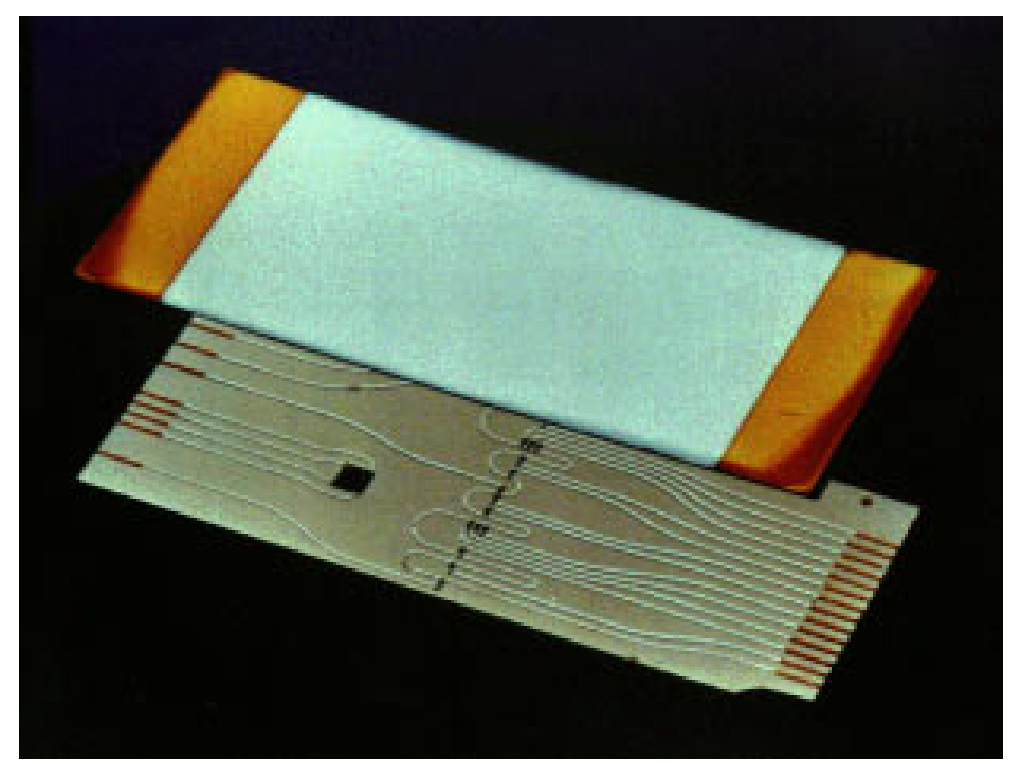

Figure 2: Final construction of the autocorrelator. 
The readout electronics, a 16-channel module, integrate, store and time-multiplex the data into a $300 \mathrm{~m}$ transmission cable (see Ref. [8]).

\subsection{Results obtained}

The evaluation of the performances with a laser has been very encouraging. The resolution of the autocorrelation of two CdTe photoconductors was 5.7 ps (sigma).

The single-shot demonstration was done by the LETI Laboratory, for an 80 ps pulse, with a long delay line on ceramic.

The first prototype of a monolithic circuit on quartz substrate had only a few working channels, as several defects had developed during the 29 steps in its production. It has nevertheless been installed in LEP and led to the first observation of autocorrelation signals from short X-ray pulses. Owing to the missing channels, a pulse shape cannot be drawn. Unfortunately, the second and third prototypes broke during mounting in the LEP set-up. The third is to be repaired and a new trial carried out.

\section{SINGLE-SHOT SAMPLER}

We now describe a wide-band optoelectronic device for short electrical single-pulse sampling [9]-[11]. The technology used is derived from the one used in the optoelectronic autocorrelator developed for CERN.

\subsection{Principle of the single-shot sampler}

The device consists of a microstrip propagation line integrated on the same sapphire substrate, normally associated with ultra-fast photoconductive switches (Fig. 3).

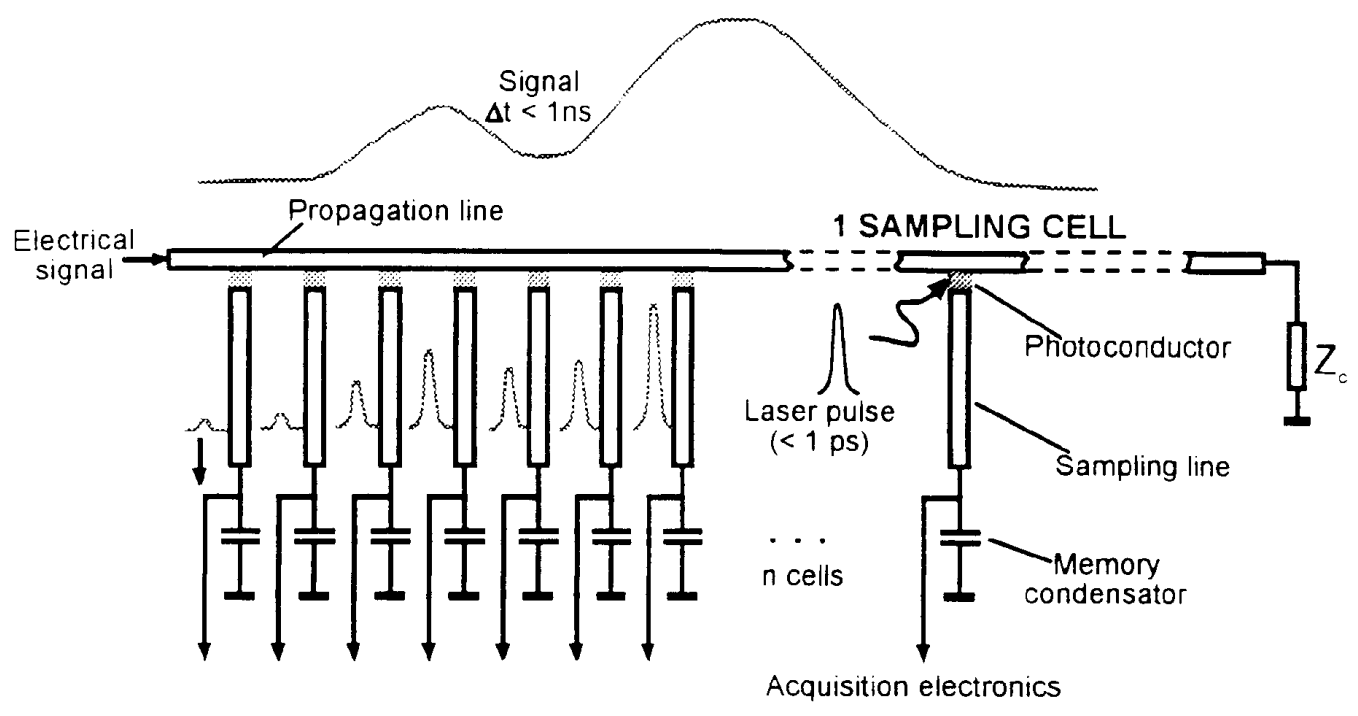

Figure 3: Principle of the optoelectronic sampler.

The electrical signal is sent in the propagation line. When the signal is entirely distributed along the line a sub-picosecond laser pulse illuminates all the photoconductors simultaneously. Thus they switch 'on' during the light pulse and every sampling line propagates a charge induced by the electric field at the sampling point. The memory capacitors store the charge of each sampling line. Morever, an electronic readout allows the reading of the charge in each memory capacitor, so that the measurement can be 
digitized and stored. Further reconstruction and display of the input electrical signal is possible.

\subsection{Semiconductor performances}

Here the very fast layers of photoconductors are used as switches. When they are illuminated their impedance decreases to some $\mathrm{k} \Omega$. In the autocorrelator described in the previous paragraph, the photoconductors are used in a linear regime and their impedance is always about twenty times higher than the line impedance. The selection of geometrical dimensions of the gap and the power of the light source fixes the type of function.

In both cases the photoconductor must have high dark resistivity, high carrier mobility, and a low carrier lifetime. Sample studies were carried out with MOCVD CdTe films and low-temperature, MBE-deposited GaAs and CdTe films [10].

A very low carrier lifetime down to 800 fs was measured on optimized MBE CdTe and GaAs films. The mobility obtained for CdTe is $<1\left(\mathrm{~cm}^{2} / \mathrm{V} \mathrm{s}\right)$ when the dark resistivity is $310^{7}(\Omega \mathrm{cm})$. The GaAs films have a mobility of 20 to $55\left(\mathrm{~cm}^{2} / \mathrm{V} \mathrm{s}\right)$ and a dark resistivity of about $10^{6}(\Omega \mathrm{cm})$.

\section{CONCLUSION}

An optoelectronic sampling system for physical instrumentation has been simulated, characterized, manufactured, and tested. It has allowed the measurement of a single electrical transient of less than 50 ps. A system capable of measuring a transient of $10 \mathrm{ps}$ in a single shot is under development in LETI.

Moreover, this optosampler could easily be adapted for single-shot measurement in the shape of new-generation, laser-induced, ultrashort X-ray pulses. Suppose a single photoconductor deposited on the delay line is irradiated by the source to be analysed and the other photoconductors sample the signal along the line. If the other photoconductors are illuminated by the source to be measured, the output is the auto-correlation signal, but if they are switched on by a very short laser probe, then the output is the sampled shape of the pulse to be measured. The CERN autocorrelator is a good example of this application for very high intensity X-ray.

This type of detector works over a very large range of X-ray, UV and visible light sources. Moreover, having demonstrated performances for single-shot electric signal analysis, we could expect in the future large new fields of application.

\section{ACKNOWLEDGMENTS}

We wish to thank C. Bovet from CERN for his continuous support, and also D. Cocq, A. Manarin, B. Jenny, J. Spanggaard, M. Sillanoli for the hardware, and G. Morpurgo for the software, of the CERN detector. We also wish to thank V. Gerbe for his basic work on the optical sampler, B. Jecko and his group at IRCOM for theoretical studies, M.-Cl. Gentet at LETI/SYS and the LETI/SLIR team for deposition and technology and M. Nail from the CEA/DAM and his team for laser experimentation. 


\section{References}

[1] M. Richter and P. Siffert, Nucl. Instrum. Methods Phys. Res. A322 (1992) 529.

[2] M. Cuzin, C. Pierrat and E. Rossa, Nucl. Instrum. Methods Phys. Res. A283 (1989) 310.

[3] E. Rossa, in Proc. SPIE 836 (1987) 22-30.

[4] E. Rossa, C. Bovet, B. Jenny, J. Spanggaard, E. Jeanclaude, M. Cuzin, M.C. Gentet and C. Ravel in Proc. 2nd European Particle Accelerator Conference (EPAC), Nice, 1990 (Edition Frontières, Gif-sur-Yvette, 1990) pp. 768-770.

[5] C. Bovet and E. Rossa, in Workshop on Advanced Beam Instrumentation, KEK, Tsukuba Japan, 1991.

[6] V.A.J. van Lint and T.M. Flanagan, Radiat. Eff. Mat. vol. 1 (John Wiley, New York, 1980).

[7] S.H. Face, C.A. Eklund and Th.A. Stringer, IEEE Trans. Nucl. Sci. NS30 No. 6 (1983) 4450.

[8] D. Cocq, A. Manarin and E. Rossa at this conference.

[9] V. Gerbe, M.C. Gentet, J. Lajzerowicz and M.Cuzin, Ultrafast Phenomena VIII (Springer Verlag, 1992) p. 145.

[10] V. Gerbe, 'Etude et réalisation d'un dispositif optoélectronique d'échantillonnage pour l'analyse d'impulsions brèves et uniques' (Thesis, Grenoble University, France, 1993).

[11] V. Gerbe, J. Lajzerowicz, M. Cuzin, L. Armengaud, M. Lalande, B. Jecko, M. Nail, Ph. Gibert, J.L. Bourgade and D. Schirmann, in Proc. SPIE 2273 (1994) 150-155. 